\title{
Vibration of Gold Nano-Beam with Variable Young's Modulus Due to Thermal Shock
}

\author{
Eman A. N. Al-Lehaibi', Hamdy M. Youssef² \\ ${ }^{1}$ Mathematics Department, College of Science and Arts-Sharoura, Najran University, Najran, KSA \\ ${ }^{2}$ Mechanics Department, Faculty of Engineering, Umm Al-Qura University, Makkah, KSA \\ Email: sa1993@hotmail.com, youssefanne2005@gmail.com
}

Received 16 November 2015; accepted 21 December 2015; published 24 December 2015

Copyright @ 2015 by authors and Scientific Research Publishing Inc.

This work is licensed under the Creative Commons Attribution International License (CC BY).

http://creativecommons.org/licenses/by/4.0/

(c) (i) Open Access

\begin{abstract}
In this paper, we will study the most important effects in the nano-scale resonator: the coupling effect of temperature and strain rate, and the non-Fourier effect in heat conduction. A solution for the generalized thermoelastic vibration of nano-resonator induced by thermal loading has been developed. The Young's modulus is taken as a linear function of the reference temperature. The effects of the thermal loading and the reference temperature in all the studied fields have been studied and represented in graphs with some comparisons. The Young's modulus makes significant effects on all the studied fields where the values of the temperature, the vibration of the deflection, stress, displacement, strain, stress-strain energy increase when the Young's modulus has taken to be variable.
\end{abstract}

\section{Keywords}

Thermoelasticity, Euler-Bernoulli Equation, Goldnano-Beam, Young's Modulus

\section{Introduction}

Diao et al. [1] were the first who discussed the effects of the free surfaces on the structure and the elastic properties of the gold nanowires by atomistic simulations. Although the atomistic simulation is a good method to calculate the elastic parameters of the nano-structured materials, it is only used to homogeneous nano-structured materials (e.g., nano-plates, nano-wires, nano-beams, ... , etc.) with a finite number of atoms.

Recently, nano mechanical resonators have attracted considerable attention due to their many applications on technology. The analysis of various effects on the characteristics of resonators, such as the resonant frequencies and the quality factorsis crucial for designing high-performance components. Many authors have studied the vibration and the heat transfer process of nano-beams [2]-[8]. Kidawa [2] studied a problem of transverse vibra- 
tions of a beam induced by a mobile heat source. The analytical solution of the problem was obtained by using the Green's functions method. While, Kidawa did not consider the thermoelastic coupling effect between the governing equations. Boley [3] studied the vibrations of a simply supported rectangular nano-beam affected by a thermal shock distributed along its span. Manolis and Beskos [4] discussed the thermally induced vibration of structures consisting of nano-beams, exposed to rapid surface heating. They have also studied the effects of the damping and the axial loads on the structural response. Al-Huniti et al. [5] investigated the thermally induced displacements and stresses of a rod using the Laplace transforms technique. Ai Kah Soh et al. studied the vibration of micro/nano-scale beam resonators induced by ultra-short-pulsed laser by considering the thermoelastic coupling term in [6] and [7]. The propagation characteristics of the longitudinal wave in nano-plates with smallscale effects are studied by Wang et al. [8].

\section{Variable Young's Modulus}

The temperature dependence of the Young's modulus for some materials was measured in the range of 293K and $973 \mathrm{~K}$, using the impulse excitation method and compared with literature data reported. The data could be fitted with [9]

$$
E=E_{0}-B T \mathrm{e}^{\left(-T_{0} / T\right)} .
$$

The values of parameters $E_{0}$ and $T_{0}$ are related to the temperature and the parameter $B$ to the harmonic character of the medium.

Farraro and Rex found that no departure from linearity was detected when they studied the dependency of the Young's modulus on the temperature, and the get the linear relation [10]

$$
E=E_{0}-E_{1} T .
$$

where $E_{0}$ is the Young's modulus in the standard case and $E_{1}$ is constant, and they measured it for pure Nickel, Platinum, and Molybdenum.

Now, we will consider the Young's modulus depends on the temperature by the following function

$$
E(T) \approx E_{0}\left(1-\gamma T_{0}\right)=E_{0} E^{*},
$$

where $\gamma$ is constant and

$$
E^{*}=\left(1-\gamma T_{0}\right)
$$

In this paper, the non-Fourier effect on heat conduction, and the coupling effect between temperature and strain rate in the nano-scale beam will be studied when Young's modulus is variable as a function of temperature. A general solution for the generalized thermoelastic vibration of gold nano-beam resonator induced by thermal shock will be developed. Laplace transforms and direct method will be used to get the lateral vibration, the temperature, the displacement, the stress-strain energy of the beam. The effects of Young's modulus will be studied and represented graphically.

\section{Problem Formulation}

Since nano-beams with rectangular cross-sections are easier to fabricate, such cross-sections are commonly adopted in the design of NEMS resonators. Consider small flexural deflections of a thin elastic beam of length $\ell(0 \leq x \leq \ell)$, width $b\left(-\frac{b}{2} \leq y \leq \frac{b}{2}\right)$ and thickness $h\left(-\frac{h}{2} \leq z \leq \frac{h}{2}\right)$, for which the $\mathrm{x}, \mathrm{y}$ and $\mathrm{z}$-axes are defined along the longitudinal, width and thickness directions of the beam, respectively (Figure 1). In equilibrium, the beam is unstrained, unstressed, without damping mechanism, and the temperature is $T_{0}$ everywhere [6].

In the present work, the Euler-Bernoulli equation is considered, and then, any plane cross-section, initially perpendicular to the axis of the beam remains plane and perpendicular to the neutral surface during bending. Thus, the displacements are given by [6] [7]:

$$
u=-z \frac{\partial w(x, t)}{\partial x}, v=0, w(x, y, z, t)=w(x, t) .
$$




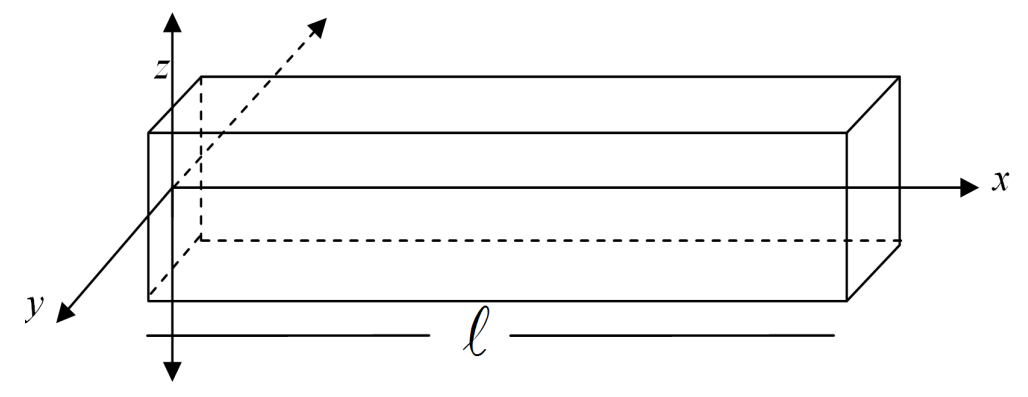

Figure 1. Cross-sections in the design of NEMS resonators.

Thus, the differential equation of thermally induced lateral vibration of the beam may be expressed in the form [6] [7] [11]-[13]:

$$
\frac{\partial^{4} w}{\partial x^{4}}+\frac{\rho A}{E_{0} E^{*} I} \frac{\partial^{2} w}{\partial t^{2}}+\alpha_{T} \frac{\partial^{2} M_{T}}{\partial x^{2}}=0
$$

where $\rho$ the density of the beam, $E$ is Young's modulus, $I\left[=b h^{3} / 12\right]$ the inertial moment about $x$-axis, $\alpha_{T}$ the coefficient of linear thermal expansion, $w(x, t)$ the lateral deflection, $x$ the distance along the length of the beam, $A=h b$ is the area of the cross section and the time and $M_{T}$ is the thermal moment as follows [6] [7] [11]-[13]:

$$
M_{T}=\frac{12}{h^{3}} \int_{-h / 2}^{h / 2} \theta z \mathrm{dz},
$$

where $\theta=\left(T-T_{0}\right)$ is the dynamical temperature increment of the resonator, $T(x, z, t)$ is the temperature distribution, and $T_{0}$ the room temperature.

According to Lord-Shulman model (L-S), the non-Fourier heat conduction equation has the following form [6] [7] [11]-[14]:

$$
\frac{\partial^{2} \theta}{\partial x^{2}}+\frac{\partial^{2} \theta}{\partial z^{2}}=\left(\frac{\partial}{\partial t}+\tau_{o} \frac{\partial^{2}}{\partial t^{2}}\right)\left(\frac{\rho C_{v}}{K} \theta+\frac{\beta T_{0}}{K} e\right)
$$

Where $e=\frac{\partial u}{\partial x}+\frac{\partial v}{\partial y}+\frac{\partial w}{\partial z}$ is the volumetric strain, $C_{v}$ is the specific heat at constant volume, $\tau_{0}$ the thermal relaxation time, $K$ the thermal conductivity, $\beta=\frac{E_{0} E^{*} \alpha_{T}}{1-2 v}$ and $v$ is Poisson's ratio. Where there is no heat flow across the upper and lower surfaces of the beam, so that $\frac{\partial \theta}{\partial z}=0$ at $z= \pm h / 2$ For a very thin nano-beam and assuming the temperature varies in terms of a $\sin (p z)$ function along the thickness direction [6] [7] [11]-[13], where $p=\pi / h$, gives

$$
\theta(x, z, t)=\theta_{1}(x, t) \sin (p z)
$$

Hence, Equation (6) gives

$$
\frac{\partial^{4} w}{\partial x^{4}}+\frac{\rho A}{E_{0} E^{*} I} \frac{\partial^{2} w}{\partial t^{2}}+\frac{12 \alpha_{T}}{h^{3}} \frac{\partial^{2} \theta_{1}}{\partial x^{2}} \int_{-h / 2}^{h / 2} z \sin (p z) \mathrm{d} z=0
$$

Moreover, Equation (8) gives

$$
\frac{\partial^{2} \theta_{1}}{\partial x^{2}} \sin (p z)-p^{2} \theta_{1} \sin (p z)=\left(\frac{\partial}{\partial t}+\tau_{o} \frac{\partial^{2}}{\partial t^{2}}\right)\left(\frac{\rho C_{v}}{K} \theta_{1} \sin (p z)-\frac{\beta T_{0}}{K} z \frac{\partial^{2} w}{\partial x^{2}}\right)
$$

After doing the integrations, Equation (10) takes the form 


$$
\frac{\partial^{4} w}{\partial x^{4}}+\frac{\rho A}{E_{0} E^{*} I} \frac{\partial^{2} w}{\partial t^{2}}+\frac{24 \alpha_{T}}{h \pi^{2}} \frac{\partial^{2} \theta_{1}}{\partial x^{2}}=0
$$

In Equation (11), we multiply the both sides by $z$ and integrating with respect to $z$ from $-\frac{h}{2}$ to $\frac{h}{2}$, and then we obtain

$$
\left(\frac{\partial^{2} \theta_{1}}{\partial x^{2}}-p^{2} \theta_{1}\right)=\left(\frac{\partial}{\partial t}+\tau_{o} \frac{\partial^{2}}{\partial t^{2}}\right)\left(\varepsilon \theta_{1}-\frac{\beta T_{0} \pi^{2} h}{24 K} \frac{\partial^{2} w}{\partial x^{2}}\right),
$$

where $\varepsilon=\frac{\rho C_{v}}{K}$.

For simplicity, we will use the following dimensionless variables [15]:

$$
\left(x^{\prime}, w^{\prime}, h^{\prime}\right)=\varepsilon c_{o}(x, w, h),\left(t^{\prime}, \tau_{o}^{\prime}\right)=\varepsilon c_{o}^{2}\left(t, \tau_{o}\right), \sigma^{\prime}=\frac{\sigma}{E_{0}}, \theta_{1}^{\prime}=\frac{\theta_{1}}{T_{o}}, c_{o}^{2}=\frac{E_{0}}{\rho} .
$$

Then, we have

$$
\frac{\partial^{4} w}{\partial x^{4}}+A_{1} \frac{\partial^{2} w}{\partial t^{2}}+A_{2} \frac{\partial^{2} \theta_{1}}{\partial x^{2}}=0
$$

and

$$
\frac{\partial^{2} \theta_{1}}{\partial x^{2}}-A_{3} \theta_{1}=\left(\frac{\partial}{\partial t}+\tau_{o} \frac{\partial^{2}}{\partial t^{2}}\right)\left(\theta_{1}-A_{4} \frac{\partial^{2} w}{\partial x^{2}}\right)
$$

where

$$
A_{1}=\frac{12}{E^{*} h^{2}}, \quad A_{2}=\frac{24 \alpha_{t} T_{0}}{\pi^{2} h}, A_{3}=p^{2}, A_{4}=\frac{\pi^{2} \beta h}{24 K \varepsilon} .
$$

For convenience, we dropped the prime.

\section{Formulation the Problem in the Laplace Transform Domain}

Applying the Laplace transform for Equations (14) and (15), this is defined by the following formula

$$
\bar{f}(s)=L[f(t)]=\int_{0}^{\infty} f(t) \mathrm{e}^{-s t} \mathrm{~d} t .
$$

Hence, we obtain the following system

$$
\frac{\mathrm{d}^{4} \bar{w}}{\mathrm{~d} x^{4}}+A_{1} \mathrm{~s}^{2} \bar{w}+A_{2} \frac{\mathrm{d}^{2} \bar{\theta}_{1}}{\mathrm{~d} x^{2}}=0
$$

and

$$
\frac{\mathrm{d}^{2} \bar{\theta}_{1}}{\mathrm{~d} x^{2}}-A_{3} \bar{\theta}_{1}=\left(s+\tau_{o} s^{2}\right)\left(\bar{\theta}_{1}-A_{4} \frac{\mathrm{d}^{2} \bar{w}}{\mathrm{~d} x^{2}}\right) .
$$

We will consider the function $\bar{\eta}$ as follows:

$$
\frac{\mathrm{d}^{2} \bar{w}}{\mathrm{~d} x^{2}}=\bar{\eta},
$$

Then, we have

$$
\frac{\mathrm{d}^{2} \overline{\theta_{1}}}{\mathrm{~d} x^{2}}=\alpha_{1} \bar{\theta}_{1}-\alpha_{2} \bar{\eta}
$$


and

$$
\frac{\mathrm{d}^{2} \bar{\eta}}{\mathrm{d} x^{2}}=-\alpha_{3} \bar{w}-\alpha_{4} \bar{\theta}_{1}+\alpha_{5} \bar{\eta},
$$

where $\alpha_{1}=\left(A_{3}+s+\tau_{o} s^{2}\right), \quad \alpha_{2}=A_{4}\left(s+\tau_{o} s^{2}\right), \quad \alpha_{3}=A_{1} s^{2}, \quad \alpha_{4}=A_{2}\left(A_{3}+s+\tau_{o} s^{2}\right), \quad \alpha_{5}=A_{2} A_{4}\left(s+\tau_{o} s^{2}\right)$.

Consider the first end of the beam $x=0$ is clamped and loaded thermally, which gives [6] [7]:

$$
w(0, t)=\eta(0, t)=0,
$$

and

$$
\theta_{1}(0, t)=\theta_{0} f(t)
$$

where $\theta_{0}$ is constant.

By using Laplace transform, the conditions will take the forms

$$
\bar{w}(0, s)=\bar{\eta}(0, s)=0 \text {, }
$$

and

$$
\bar{\theta}_{1}(0, s)=\theta_{0} \bar{f}(s) .
$$

Consider the other end of the beam $x=\ell$ is clamped and remains at zero increments of temperature as follows:

$$
w(\ell, t)=\theta_{1}(\ell, t)=\eta(\ell, t)=0 .
$$

After using Laplace transform, we have

$$
\bar{w}(\ell, s)=\bar{\theta}_{1}(\ell, s)=\bar{\eta}(\ell, s)=0 .
$$

After some simplifications by using MAPLE programme, we get the final solutions in the Laplace transform domain as follows:

The lateral deflection

$$
\begin{aligned}
\bar{w}(x, s)= & \frac{\Delta \sinh \left(k_{1}(\ell-x)\right)}{\left(k_{1}^{2}-k_{2}^{2}\right)\left(k_{1}^{2}-k_{3}^{2}\right) \sinh \left(k_{1} \ell\right)}+\frac{\Delta \sinh \left(k_{2}(\ell-x)\right)}{\left(k_{2}^{2}-k_{1}^{2}\right)\left(k_{2}^{2}-k_{3}^{2}\right) \sinh \left(k_{2} \ell\right)} \\
& +\frac{\Delta \sinh \left(k_{3}(\ell-x)\right)}{\left(k_{3}^{2}-k_{1}^{2}\right)\left(k_{3}^{2}-k_{2}^{2}\right) \sinh \left(k_{3} \ell\right)} .
\end{aligned}
$$

The temperature

$$
\begin{aligned}
\bar{\theta}(z, x, s)= & -\frac{\alpha_{2} k_{1}^{2} \Delta \sin (p z) \sinh \left(k_{1}(\ell-x)\right)}{\left(k_{1}^{2}-\alpha_{1}\right)\left(k_{1}^{2}-k_{2}^{2}\right)\left(k_{1}^{2}-k_{3}^{2}\right) \sinh \left(k_{1} \ell\right)}-\frac{\alpha_{2} k_{2}^{2} \Delta \sin (p z) \sinh \left(k_{2}(\ell-x)\right)}{\left(k_{2}^{2}-\alpha_{1}\right)\left(k_{2}^{2}-k_{1}^{2}\right)\left(k_{2}^{2}-k_{1}^{2}\right) \sinh \left(k_{2} \ell\right)} \\
& -\frac{\alpha_{3} k_{2}^{2} \Delta \sin (p z) \sinh \left(k_{3}(\ell-x)\right)}{\left(k_{3}^{2}-\alpha_{1}\right)\left(k_{3}^{2}-k_{1}^{2}\right)\left(k_{3}^{2}-k_{2}^{2}\right) \sinh \left(k_{3} \ell\right)} .
\end{aligned}
$$

The displacement

$$
\begin{aligned}
\bar{u}(z, x, s)= & -\frac{z \Delta k_{1} \cosh \left(k_{1}(\ell-x)\right)}{\left(k_{1}^{2}-k_{2}^{2}\right)\left(k_{1}^{2}-k_{3}^{2}\right) \sinh \left(k_{1} \ell\right)}-\frac{z \Delta k_{2} \cosh \left(k_{2}(\ell-x)\right)}{\left(k_{2}^{2}-k_{1}^{2}\right)\left(k_{2}^{2}-k_{1}^{2}\right) \sinh \left(k_{2} \ell\right)} \\
& -\frac{z \Delta k_{3} \cosh \left(k_{3}(\ell-x)\right)}{\left(k_{3}^{2}-k_{1}^{2}\right)\left(k_{3}^{2}-k_{2}^{2}\right) \sinh \left(k_{3} \ell\right)} .
\end{aligned}
$$

The Strain 


$$
\begin{aligned}
\bar{e}(z, x, s)= & \frac{z \Delta k_{1}^{2} \sinh \left(k_{1}(\ell-x)\right)}{\left(k_{1}^{2}-k_{2}^{2}\right)\left(k_{1}^{2}-k_{3}^{2}\right) \sinh \left(k_{1} \ell\right)}+\frac{z \Delta k_{2}^{2} \sinh \left(k_{2}(\ell-x)\right)}{\left(k_{2}^{2}-k_{1}^{2}\right)\left(k_{2}^{2}-k_{1}^{2}\right) \sinh \left(k_{2} \ell\right)} \\
& +\frac{z \Delta k_{3}^{2} \sinh \left(k_{3}(\ell-x)\right)}{\left(k_{3}^{2}-k_{1}^{2}\right)\left(k_{3}^{2}-k_{2}^{2}\right) \sinh \left(k_{3} \ell\right)} .
\end{aligned}
$$

where

$$
\begin{gathered}
\Delta=\frac{\theta_{0} \bar{f}(s)}{\alpha_{1} \alpha_{2}}\left(\alpha_{1}-k_{1}^{2}\right)\left(\alpha_{1}-k_{2}^{2}\right)\left(\alpha_{1}-k_{3}^{2}\right) \text { and } \pm k_{1}, \pm k_{2}, \pm k_{3} \text { are the roots of the equation } \\
\qquad k^{6}-l k^{4}+m k^{2}-n=0,
\end{gathered}
$$

and

$l=\alpha_{1}+\alpha_{5}, \quad m=\alpha_{1} \alpha_{5}-\alpha_{2} \alpha_{4}+\alpha_{3}$ and $n=\alpha_{1} \alpha_{3}$.

\section{The Stress-Strain Energy}

The stress on the $x$-axis, according to Hooke's law is:

$$
\sigma_{x x}(x, z, t)=E_{0} E^{*}\left(e-\alpha_{T} \theta\right) .
$$

By using the non-dimensional variables in (13), we obtain the stress in the form

$$
\sigma_{x x}(x, z, t)=E^{*}\left(e-\alpha_{T} T_{0} \theta\right) .
$$

By using Laplace transform, the above equation takes the form:

$$
\bar{\sigma}_{x x}(x, z, s)=E^{*}\left(\bar{e}-\alpha_{T} T_{0} \bar{\theta}\right) .
$$

The stress-strain energy, which is generated by the beam, is given by

$$
W(x, z, t)=\sum_{i, j=1}^{3} \frac{1}{2} \sigma_{i j} e_{i j}=\frac{1}{2} \sigma_{x x} e_{x x}=-\frac{1}{2} z \sigma_{x x} \eta,
$$

We can re-write Equation (36) to be in the form

$$
W(x, z, t)=-\frac{1}{2} z\left[L^{-1}\left(\bar{\sigma}_{x x}\right)\right]\left[L^{-1}(\bar{\eta})\right],
$$

$L^{-1}[\bullet]$ is the inversion of Laplace transform.

To complete the solution in the Laplace transform domain, we have to determine the type of heating which we have used to load the boundary of the medium thermally.

We have applied harmonic thermal loading as follows [16]:

$$
f(t)=\sin (\omega t) \text {, }
$$

after using Laplace transform, we obtain

$$
\bar{f}(s)=\frac{\omega}{s^{2}+\omega^{2}},
$$

$\omega$ is the angular frequency of thermal vibration.

\section{Numerical Inversion of the Laplace Transform}

To determine the solutions in the time domain, the Riemann-sum approximation method is used to obtain the numerical results. In this method, any function in Laplace domain can be inverted to the time domain as

$$
f(t)=\frac{\mathrm{e}^{\kappa t}}{t}\left[\frac{1}{2} \bar{f}(\kappa)+R e \sum_{n=1}^{N}(-1)^{n} \bar{f}\left(\kappa+\frac{i n \pi}{t}\right)\right]
$$


where $R e$ is the real part and $i$ is imaginary number unit. For faster convergence, numerous numerical experiments have shown that the value of $\kappa$ satisfies the relation $\kappa t \approx 4.7 \quad$ Tzou [17].

\section{Numerical Results and Discussion}

Now, we will consider a numerical example for which computational results are given. For this purpose, Gold $\mathrm{Au}$ is taken as the thermoelastic material for which we take the following values of the different physical constants [18]:

$$
\begin{aligned}
& \quad k=318 \mathrm{~W} /(\mathrm{m} \cdot \mathrm{K}), \quad \alpha_{T}=14.2 \times(10)^{-6} \mathrm{~K}^{-1}, \rho=1930 \mathrm{~kg} / \mathrm{m}^{3}, T_{0}=293 \mathrm{~K}, C_{v}=130 \mathrm{~J} /(\mathrm{kg} \cdot \mathrm{K}), \\
& E=180 \mathrm{GPa}, \quad v=0.44 .
\end{aligned}
$$

The aspect ratios of the beam are fixed as $\ell / h=10$ and $b / h=1 / 2$ when $h$ is varied, $\ell$ and $b$ change accordingly with $h$.

For the nano-scale beam, we will take the range of the beam length $\ell(1-100) \times 10^{-9} \mathrm{~m}$. The original time $t$ will be considered in the picoseconds $(1-100) \times 10^{-12}$ sec and the relaxation time $\tau_{0}$ in the range $(1-100) \times 10^{-14}$ sec .

The figures (Figure 2-7) were prepared by using the non-dimensional variables which are defined in (9) for beam length $\ell=1.0, \theta_{0}=1.0 \quad \mathrm{z}=h / 6$ and $t=0.1$.

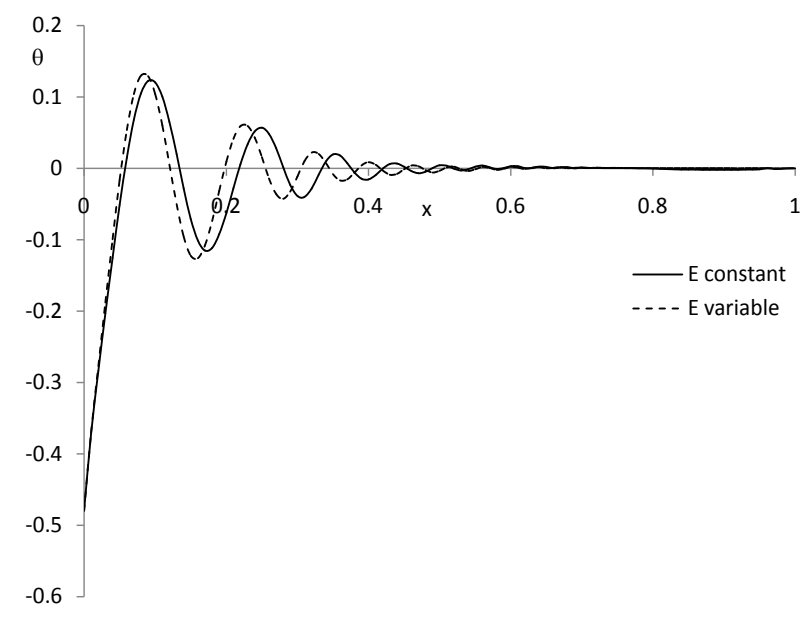

Figure 2. The temperature distribution with different cases of Young's modulus.

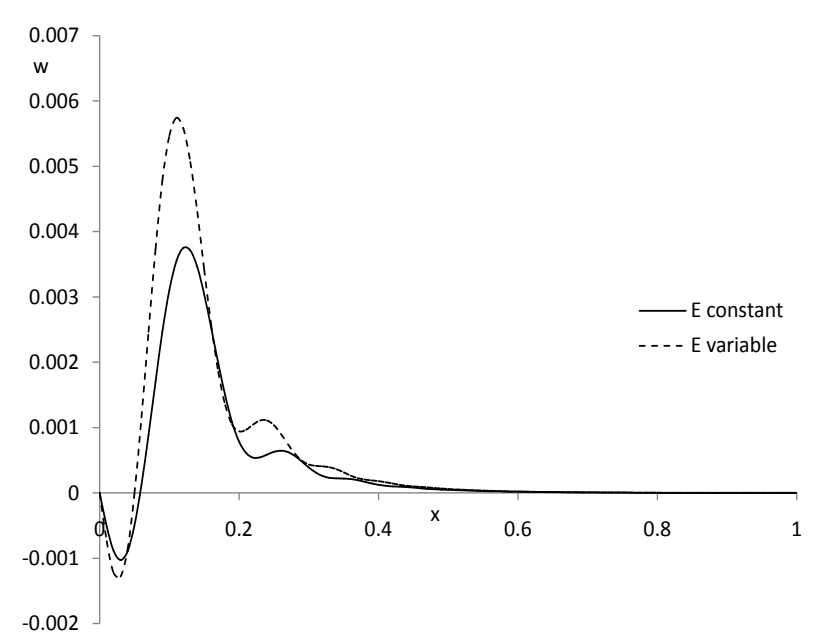

Figure 3. The lateral vibration distribution with different cases of Young's modulus. 


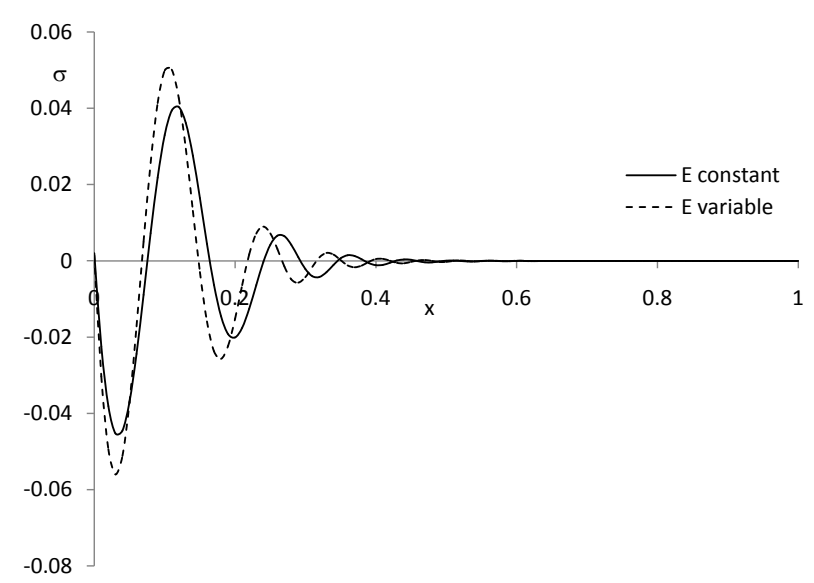

Figure 4. The stress distribution with different cases of Young's modulus.

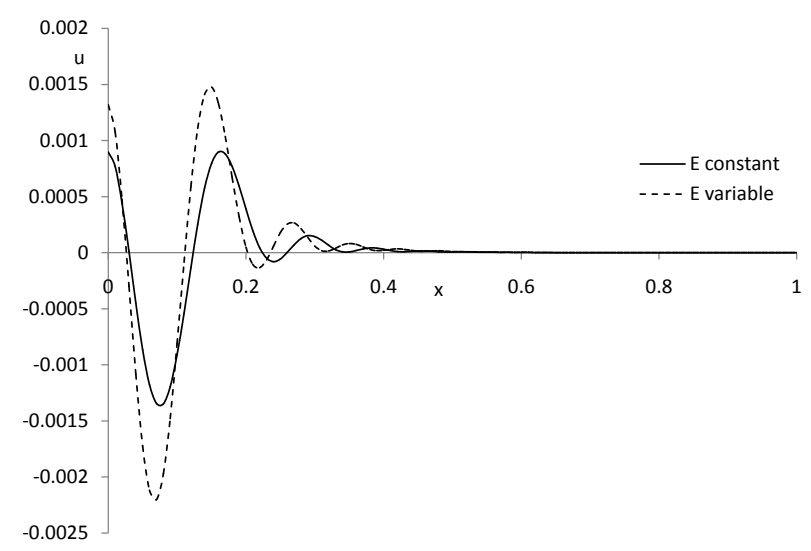

Figure 5. The displacement distribution with different cases of Young's modulus.

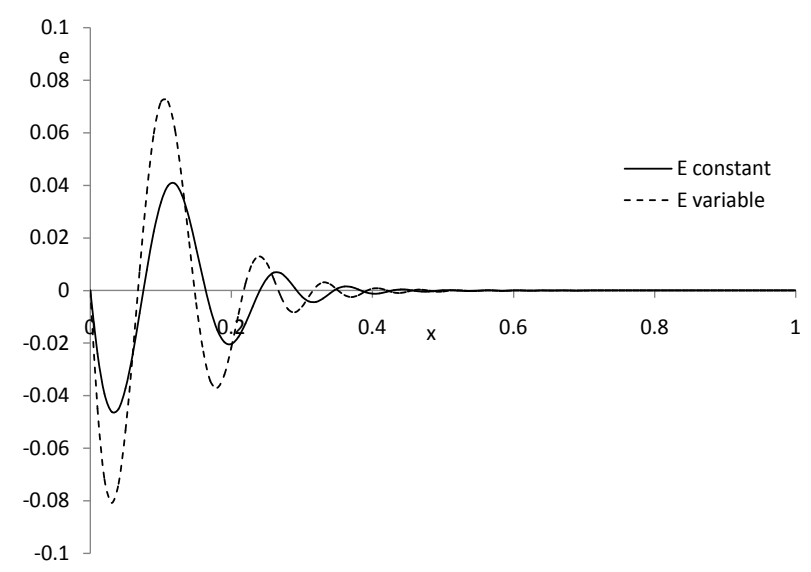

Figure 6. The strain distribution with different cases of Young's modulus.

\section{Conclusion}

The Young's modulus has significant effects on all the studied fields. The values of the temperature, the vibra- 


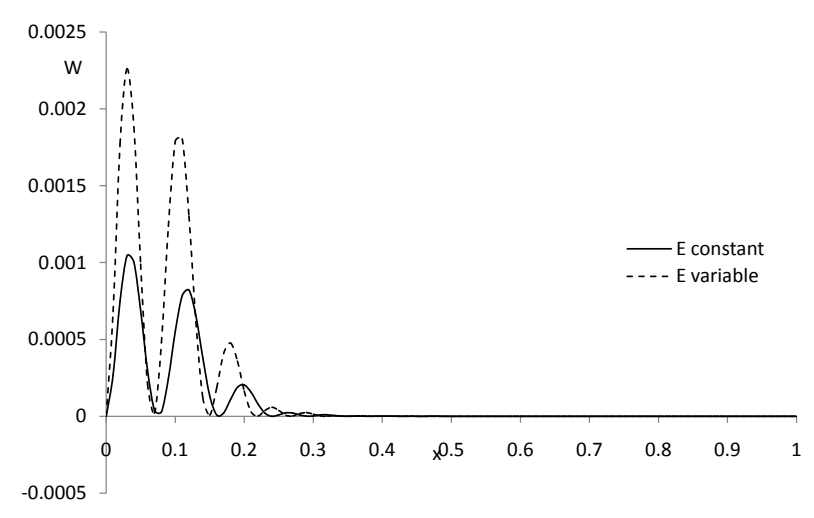

Figure 7. The stress-strainenergy distribution with different cases of Young's modulus.

tion of the deflection, stress, displacement, strain, stress-strain energy increase when the Young's modulus is variable. The peak points of all the distributions increase when the Young's modulus is variable with large differences in the case of Young's modulus is constant.

\section{References}

[1] Diao, J.K., Gall, K. and Dunn, M.L. (2004) Atomistic Simulation of the Structure and Elastic Properties of Gold Nanowires. Journal of the Mechanics and Physics of Solids, 52, 1935-1962. http://dx.doi.org/10.1016/j.jmps.2004.03.009

[2] Kidawa-Kukla, J. (2003) Application of the Green Functions to the Problem of the Thermally Induced Vibration of a Beam. Journal of Sound and Vibration, 262, 865-876. http://dx.doi.org/10.1016/S0022-460X(02)01133-1

[3] Boley, B.A. (1972) Approximate Analyzes of Thermally Induced Vibrations of Beams and Plates. Journal of Applied Mechanics, 39, 212-216. http://dx.doi.org/10.1115/1.3422615

[4] Manolis, G.D. and Beskos, D.E. (1980) Thermally Induced Vibrations of Beam Structures. Computer Methods in Applied Mechanics and Engineering, 21, 337-355. http://dx.doi.org/10.1016/0045-7825(80)90101-2

[5] Al-Huniti, N.S., Al-Nimr, M.A. and Naij, M. (2001) Dynamic Response of a Rod Due to a Moving Heat Source under the Hyperbolic Heat Conduction Model. Journal of Sound and Vibration, 242, 629-640. http://dx.doi.org/10.1006/jsvi.2000.3383

[6] Soh, A.K., Sun, Y.X. and Fang, D.N. (2008) Vibration of Microscale Beam Induced by Laser Pulse. Journal of Sound and Vibration, 311, 243-253. http://dx.doi.org/10.1016/j.jsv.2007.09.002

[7] Sun, Y.X., Fang, D.N., Saka, M. and Soh, A.K. (2008) Laser-Induced Vibrations of Micro-Beams under Different Boundary Conditions. International Journal of Solids and Structures, 45, 1993-2013. http://dx.doi.org/10.1016/j.ijsolstr.2007.11.006

[8] Wang, Y.Z., Li, F.M. and Kishimoto, K. (2010) Scale Effects on the Longitudinal Wave Propagation in Nanoplates. Physica E, 42, 1356-1360. http://dx.doi.org/10.1016/j.physe.2009.11.036

[9] Bruls, R.J., Hintzen, H.T., De With, G. and Metselaar, R. (2001) The Temperature Dependence of the Young’s Modulus of MgSiN2, AlN and Si3N4. Journal of the European Ceramic Society, 21, 263-268. http://dx.doi.org/10.1016/S0955-2219(00)00210-7

[10] Farraro, R. and McLellan, R.B. (1977) Temperature Dependence of the Young's Modulus and Shear Modulus of Pure Nickel, Platinum, and Molybdenum. Metallurgical Transactions A, 8, 1565-1563. http://dx.doi.org/10.1007/BF02644859

[11] Sun, Y.X., Fang, D.N. and Soh, A.K. (2006) Thermoelastic Damping in Micro-Beam Resonators. International Journal of Solids and Structures, 43, 3213-3229. http://dx.doi.org/10.1016/j.ijsolstr.2005.08.011

[12] Fang, D.N., Sun, Y.X. and Soh, A.K. (2006) Analysis of Frequency Spectrum of Laser-Induced Vibration of Microbeam Resonators. Chinese Physics Letters, 23, 1554-1557. http://dx.doi.org/10.1088/0256-307X/23/6/055

[13] Duwel, A., Gorman, J., Weinstein, M., Borenstein, J. and Ward, P. (2003) Experimental Study of Thermoelastic Damping in MEMS Gyros. Sensors and Actuators A, 103, 70-75. http://dx.doi.org/10.1016/S0924-4247(02)00318-7

[14] Lord, H. and Shulman, Y. (1967) A Generalized Dynamical Theory of Thermoelasticity. Journal of the Mechanics and Physics of Solids, 15, 299-309. http://dx.doi.org/10.1016/0022-5096(67)90024-5

[15] Youssef, H.M. (2013) Vibration of Gold Nano-Beam with Variable Thermal Conductivity: State-Space Approach. Ap- 
plied Nanoscience, 3, 397-407.

[16] Youssef, H.M., Elsibai, K.A. and El-Bary, A.A. (2014) Vibration of Cylindrical Gold Nano-Beam with Fractional Order Thermoelastic Waves. Jökull Journal, 64, 416-427.

[17] Tzou, D. (1996) Macro-to-Micro Heat Transfer. Taylor \& Francis, Washington DC.

[18] Youssef, H.M., Elsibai, K.A. and El-Bary, A.A. (2014) Vibration of Gold NanoBeam in Context of Two-Temperature Generalized Thermoelasticity Subjected to Laser Pulse. Latin American Journal of Solids and Structures, 11, 24602482. http://dx.doi.org/10.1590/S1679-78252014001300008 